\title{
Diamond drizzle forecast for Saturn and Jupiter
}

Lightning storms create carbon soot that might be compressed into diamonds as it falls through the atmosphere.

\section{Maggie McKee}

09 October 2013

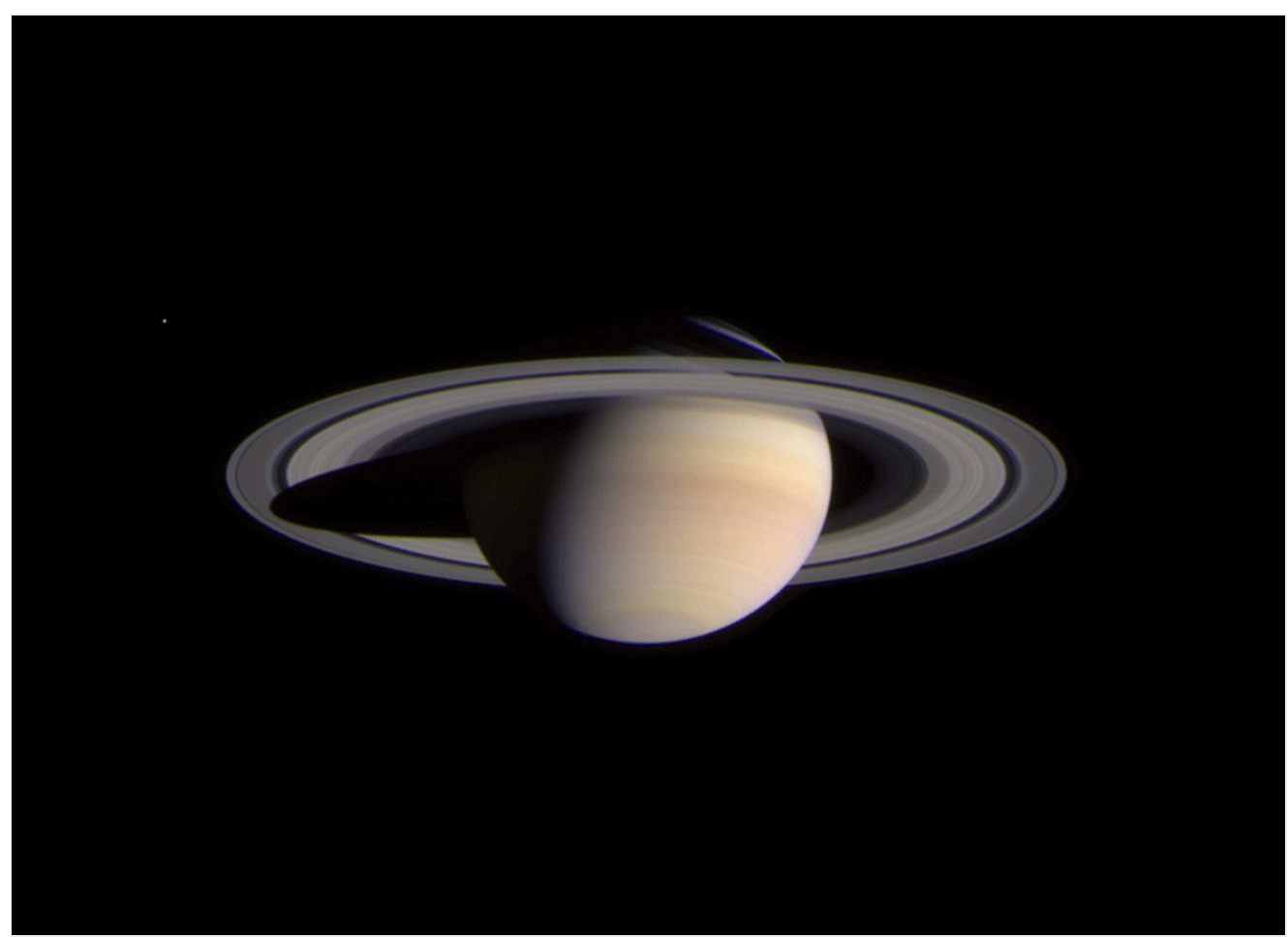

NASA/JPL/Space Science Institute

High pressures on Saturn could be squeezing soot into diamonds, which then falls as rain.

Forget diamonds in the sky - it may actually be raining diamonds on Saturn and Jupiter, according to two planetary scientists ${ }^{1}$.

Researchers have long wondered whether the high pressures inside the giant planets could turn carbon into diamond ${ }^{2}$, and even though some researchers dispute their claim, Mona Delitsky of California Specialty Engineering in Flintridge, and Kevin Baines of the University of Wisconsin-Madison, now say it is possible. They are laying out their argument this week at a meeting of the American Astronomical Society's Division for Planetary Sciences in Denver, Colorado.

In their scenario, lightning zaps molecules of methane in the upper atmospheres of Saturn and Jupiter, liberating carbon atoms. These atoms then stick onto each other, forming larger particles of carbon soot, which the Cassini spacecraft may have spotted in dark storm clouds on Saturn ${ }^{3}$. As the soot particles slowly float down through ever-denser layers of gaseous and liquid hydrogen towards the planets' rocky cores, they experience ever greater pressures and temperatures. The soot is compressed into graphite, and then into solid diamonds before reaching a temperature of about $8,000{ }^{\circ} \mathrm{C}$, when the diamond melts, forming liquid diamond raindrops, they say.

\section{Precious rain}

Inside Saturn, the conditions are right for diamond 'hail' to form, beginning at a depth of about 6,000 kilometres into the atmosphere and extending for another $30,000 \mathrm{~km}$ below that, says Baines. He estimates that Saturn may harbour about 10 million tonnes of diamond produced this way, with most of it made up of rocks no bigger than a millimetre and perhaps some chunks spanning 10 centimetres.

"If you had a robot there, it would sit there and collect diamonds raining down," Baines says. Indeed, this is just what happens in a piece of sci-fi the authors wrote to augment a recent non-fiction book chapter ${ }^{4}$ on the science of Saturn's fluid atmosphere. In their vision of the year 2469, diamonds would be collected on Saturn and used to make the ultra-strong hulls of mining ships delving deep into the planet's interior to collect helium-3 for clean-burning fusion fuel. The diamonds must stay in Saturn to prevent havoc in 
Delitsky and Baines concluded that diamond would be stable inside the giant planets by comparing recent studies that experimentally fleshed out the temperatures and pressures at which carbon takes on different forms ${ }^{5}$, such as solid diamond, and others that modelled the pressure and temperature at different depths within the giant planets ${ }^{6}$. "We put together two pieces of information from different sources to conclude that diamonds are likely to be existing in the deep interiors of Saturn and Jupiter," says Delitsky.

\section{Thermodynamic objections}

But others dispute the researchers' claim, arguing that these two gas giants of the Solar System are probably devoid of bling. David Stevenson, a planetary scientist at the California Institute of Technology in Pasadena, says the pair did not properly

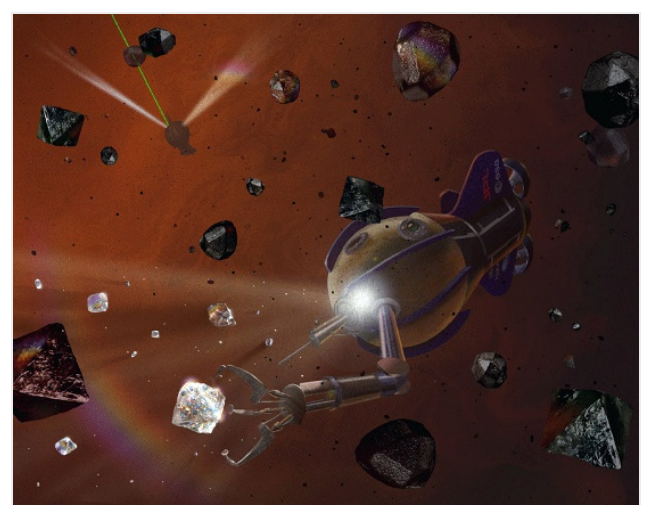

Image courtesy of Michael Camoll Alien Seas: Oceans in space (Springer 2013)

Could robots one day collect diamonds on Saturn? account for thermodynamics. Methane forms a very small fraction of the mostly hydrogen atmospheres of Jupiter and Saturn $-0.2 \%$ and $0.5 \%$, respectively. In such dilute systems, "thermodynamics favours mixtures", says Stevenson. "It's the same thermodynamics that explains why a small amount of sugar or salt will dissolve in a large amount of water, especially at high temperature," he says. "Even if you made carbon dust, it would just dissolve as it went down into the interior very very quickly."

Luca Ghiringhelli, a physicist at the Fritz Haber Institute in Berlin, Germany, is also sceptical of the pair's conclusions. His own previous research ${ }^{7}$ showed that the concentration of carbon is not high enough in Uranus and Neptune - which are several times richer in carbon than Jupiter and Saturn — to grow diamond from the 'ground up', building the crystals atom by atom. He did not model whether diamond could instead form by the compression of soot, but says it is premature to assume that would do so. "It is very optimistic to drive conclusions on the existence of diamonds on Saturn from the scarce data we have, and without a convincing model," he says. That may give financial regulators one less thing to worry about in the coming centuries.

Nature I doi:10.1038/nature.2013.13925

\section{References}

1. Delitsky, M. L. \& Baines, K. H. Bull. Am. Astron. Soc. 49, No. 9, abstract 512.09 (2013).

2. Ross, M. Nature 292, 435-436 (1981).

3. Baines, K. H. et al. Planet. Space Sci. 57, 1650-1658 (2009).

4. Baines, K. H. \& Delitsky, M. L. in Alien Seas: Oceans in Space (eds Carroll, M. \& Lopes, R.) 16-17 (2013).

5. Knudson, M. D., Desjarlais, M. P. \& Dolan, D. H. Science 322, 1822-1825 (2008).

6. Nettelmann, N. et al. Astrophys. J. 683, 1217-1228 (2008).

7. Ghiringhelli, L. M., Valeriani, C., Meijer, E. J. \& Frenkel, D. Phys. Rev. Lett. 99, 055702 (2007). 